\title{
Electron-Capture Rates of Nuclei at Stellar Environments and Nucleosynthesis
}

\author{
Toshio Suzuki ${ }^{1,5}$, Michio Honma ${ }^{2}$, Noritaka Shimizu ${ }^{3}$, Yusuke Tsunoda ${ }^{3}$, \\ Takaharu Otsuka $^{4,10}$, Toshitaka Kajino ${ }^{5,11}$, Kanji Mori ${ }^{5,11}$, Michael Famiano ${ }^{5,6}$ \\ Jun Hidaka ${ }^{5,7}$, Ken'ichi Nomoto ${ }^{8,12}$ and Koichi Iwamoto ${ }^{9}$ \\ ${ }^{I}$ Department of Physics, College of Humanities and Sciences, Nihon University \\ Sakurajosui 3-25-40, Setagaya-ku, Tokyo 156-8550, Japan \\ ${ }^{2}$ Center for Mathematical Sciences, University of Aizu, Fukushima 965-8580, Japan \\ ${ }^{3}$ Center for Nuclear Study, the University of Tokyo, Hongo, Tokyo 113-0033, Japan \\ ${ }^{4}$ Department of Physics, The University of Tokyo, Hongo, Tokyo 113-0033, Japan \\ ${ }^{5}$ National Astronomical Observatory of Japan, Mitaka, Tokyo 181-8588, Japan \\ ${ }^{6}$ Department of Physics, Western Michigan University, Michigan 49008, USA \\ ${ }^{7}$ Mechanical Engineering Department, Meisei University, Hino, Tokyo 191-8506, Japan \\ ${ }^{8}$ Kavli Institute for the Physics and Mathematics (WPI), The University of Tokyo, Kashiwa, Chiba 277- \\ 8583, Japan \\ ${ }^{9}$ Department of Physics, College of Science and Technology, Tokyo 101-8308, Japan \\ ${ }^{10}$ National Superconducting Cyclotron Laboratory, Michigan State University, MI 48824, USA \\ ${ }^{11}$ Department of Astronomy, School of Science, The University of Tokyo, Hongo, Tokyo 113-0033, Japan \\ ${ }^{12}$ Hamamatsu Professor
}

E-mail: suzuki@phys.chs.nihon-u.ac.jp, m-honma@u-aizu.ac.jp, shimizu@cns.s.u-tokyo.ac.jp, ytsunoda@nt.phys.s.u-tokyo.ac.jp, otsuka@phys.s.u-tokyo.ac.jp, kajino@nao.ac.jp, kanji.mori@nao.ac.jp. michael.famiano@wmich.edu,jun.hidaka@meisei-u.ac.jp,nomoto@astron.s.u-tokyo.ac.jp, iwamoto.kouichi@nihon-u.ac.jp

Electron-capture rates in nuclei at stellar environments are evaluated by using new shell-model Hamiltonians. A new Hamiltonian for pf-shell, GXPF1J, can describe Gamow-Teller (GT) strengths in Ni isotopes very accurately. In particular, large spreading in the GT strength in ${ }^{56} \mathrm{Ni}$ and ${ }^{55} \mathrm{Co}$ obtained for GXPF1J has been confirmed by a recent experiment .The updated e-capture rates as well as beta-decay rates obtained in a large region of pf-shell nuclei are applied to study nucleosynthesis in Type-Ia supernova explosions and core-collapse supernova explosions. The e-capture rates with GXPF1J, which are generally smaller than those with conventional shell-model Hamiltonians such as KB3G and lead to less production of neutron-rich nuclei such as ${ }^{58} \mathrm{Ni}$ and ${ }^{54} \mathrm{Cr}$, can thus solve the problem of over-production of neutron-rich isotopes in the $\mathrm{Fe}$ region compared to the solar abundance. For more neutron-rich isotopes such as ${ }^{78} \mathrm{Ni}$, extension of the configuration space outside the pf-shell is essential. Here, GT strength and spin-dipole strengths in ${ }^{78} \mathrm{Ni}$ are evaluated within $\mathrm{pf}+\mathrm{g}_{9 / 2}+\mathrm{d}_{5 / 2}$ shells. Core-collapse supernova explosions are sensitive to the e-capture rates for nuclei around this region. Electron-capture rates in ${ }^{78} \mathrm{Ni}$ are discussed with shell-model as well as with RPA calculations.

The 26th International Nuclear Physics Conference

11-16 September, 2016

Adelaide, Australia

\footnotetext{
${ }^{1}$ Speaker

(C) Copyright owned by the author(s) under the terms of the Creative Commons 


\section{Introduction}

Electron-capture rates in pf-shell nuclei at stellar environments are evaluated with new shell-model Hamiltonians, and applied to study nucleosynthesis of iron-group nuclei in Type Ia supernova explosions ( $\mathrm{SNe}$ ). Over-production of neutron-rich iron-group nuclei compared to the solar abundances obtained for the Fuller-Fowler-Newman (FFN) e-capture rates [1] is found to be reduced to within a factor of around 2 by using the new Hamiltonian, GXPF1J [2], which gives Gamow-Teller (GT) strengths consistent with experimental data in $\mathrm{Ni}$ and Fe isotopes $[3,4]$. Electron-capture rates in a pfg-shell nucleus, ${ }^{78} \mathrm{Ni}$, are also investigated by shell-model and RPA calculations as the capture rates for isotones with $\mathrm{N}=50$ are expected to affect nucleosynthesis in core-collapse $\mathrm{SNe}$ considerably [5].

In sect. 2, GT strengths in Ni isotopes obtained with GXPF1J are shown to explain the experimental data very well, and electron-capture rates at stellar environments are obtained and compared with other calculations. In sect. 3, nucleosynthesis of iron-group elements in Type Ia $\mathrm{SNe}$ is discussed comparing the results of the present rates and other shell-model rates. In sect. 3, electron-capture rates in ${ }^{78} \mathrm{Ni}$ are evaluated by shell-model calculations within $\mathrm{pf}-\mathrm{g}_{9 / 2} \mathrm{~d}_{5 / 2}$ configurations and compared with RPA calculations as well as an approximate capture formula in Ref. [5]. A summary is given in sect. 4.

\section{GT strengths in Ni isotopes and e-capture rates at stellar environments}

GT strengths in pf-shell nuclei are evaluated by GXPF1J and KB3G [6] Hamiltonians with the universal quenching factor of $\mathrm{g}_{\mathrm{A}}{ }^{\text {eff }} / \mathrm{g}_{\mathrm{A}}=0.74$ [7]. GXPF1J is modified from GXPF1A [8] to reproduce the peak energy of the main M1 strength in ${ }^{48} \mathrm{Ca}$. These shell-model Hamiltonians can describe spin degree's freedom of pf-shell nuclei quite well, and considerable improvements over FFN [1] have been achieved for the GT transition strengths. While the sum of B(GT.) values for $\mathrm{Fe}$ amd $\mathrm{Ni}$ isotopes are similar for GXPF1J and KB3G, there is a clear difference in the distribution of the GT strength between the two Hamiltonians. Here, $\mathrm{B}\left(\mathrm{GT}_{ \pm}\right)$is defined to be $\left|\left\langle\mathrm{f}\left\|\vec{\sigma} \mathrm{t}_{ \pm}\right\| \mathrm{i}\right\rangle\right|^{2} /\left(2 \mathrm{~J}_{\mathrm{i}}+1\right)$, where $\mathrm{J}_{\mathrm{i}}$ is the spin of the initial state, $\mathrm{t}_{-}|\mathrm{n}\rangle=|\mathrm{p}\rangle$ and $\mathrm{t}_{+}|\mathrm{p}\rangle=|\mathrm{n}\rangle$. The GXPF1J generally gives the strengths more spread compared to KB3G. For examle, GXPF1J explains the experimental distribution of the $\mathrm{B}\left(\mathrm{GT}_{-}\right)$in ${ }^{58} \mathrm{Ni}$ better than $\mathrm{KB} 3 \mathrm{G}[2,9]$. It is also true for $\mathrm{B}\left(\mathrm{GT}_{+}\right)$in ${ }^{58} \mathrm{Ni}$ and ${ }^{60} \mathrm{Ni}$ [3]. Electron-capture rates obtained with GXPF1A and $\mathrm{KB} 3 \mathrm{G}$ as well as RPA calculations are compared with experimental values for 13 pf-shell nuclei, and GXPF1A proves to be the best one among them [10].

We discuss especially the GT strength in ${ }^{56} \mathrm{Ni}$. Two-peak structure with large fragmentation of the strength is obtained with GXPF1J while it is not the case for other Hamiltonians such as KB3G and KBF [11] as shown in Fig. 1. The two-peak structure has been confirmed by recent $(\mathrm{p}, \mathrm{n})$ reaction experiment [12]. This leads to smaller e-capture rates for GXPF1J compared to KB3G as shown in Fig. 2 due to less strength at low excitation energy $\left(E_{x}\right)$ region. In case of $\mathrm{KBF}$, as the strength is smaller for GXPF1J at $\mathrm{E}_{\mathrm{x}}=3-4.5 \mathrm{MeV}$ but larger at $\mathrm{E}_{\mathrm{x}}=2-3 \mathrm{MeV}$ and $\mathrm{E}_{\mathrm{x}}>4.5 \mathrm{MeV}$ (see Fig. 1), order of the magnitude of calculated e-capture rates depends on the density and temperature. Note that electron chemical potential is 5.1 (2.4) $\mathrm{MeV}$ at $\rho \mathrm{Y}_{\mathrm{e}}=10^{9}$ $\left(10^{8}\right) \mathrm{g} \mathrm{cm}^{-3}$. The capture rates of GXPF1J are smaller (larger) than those of KBF at $\rho \mathrm{Y}_{\mathrm{e}}=10^{7}$ and $10^{9} \mathrm{~g} \mathrm{~cm}^{-3}\left(10^{8} \mathrm{~g} \mathrm{~cm}^{-3}\right)$ at $\mathrm{T}_{9}<3$, where $\mathrm{Y}_{\mathrm{e}}$ is the lepton to baryon ratio or proton fraction and 
$\mathrm{T}_{9}=\mathrm{T} / 10^{9}$ (see Fig. 2). Similar larger spreading for GXPF1J is also found in the GT . strength in ${ }^{55} \mathrm{Co}\left(\mathrm{GT}_{+}\right.$strength in $\left.{ }^{55} \mathrm{Ni}\right)[12]$.
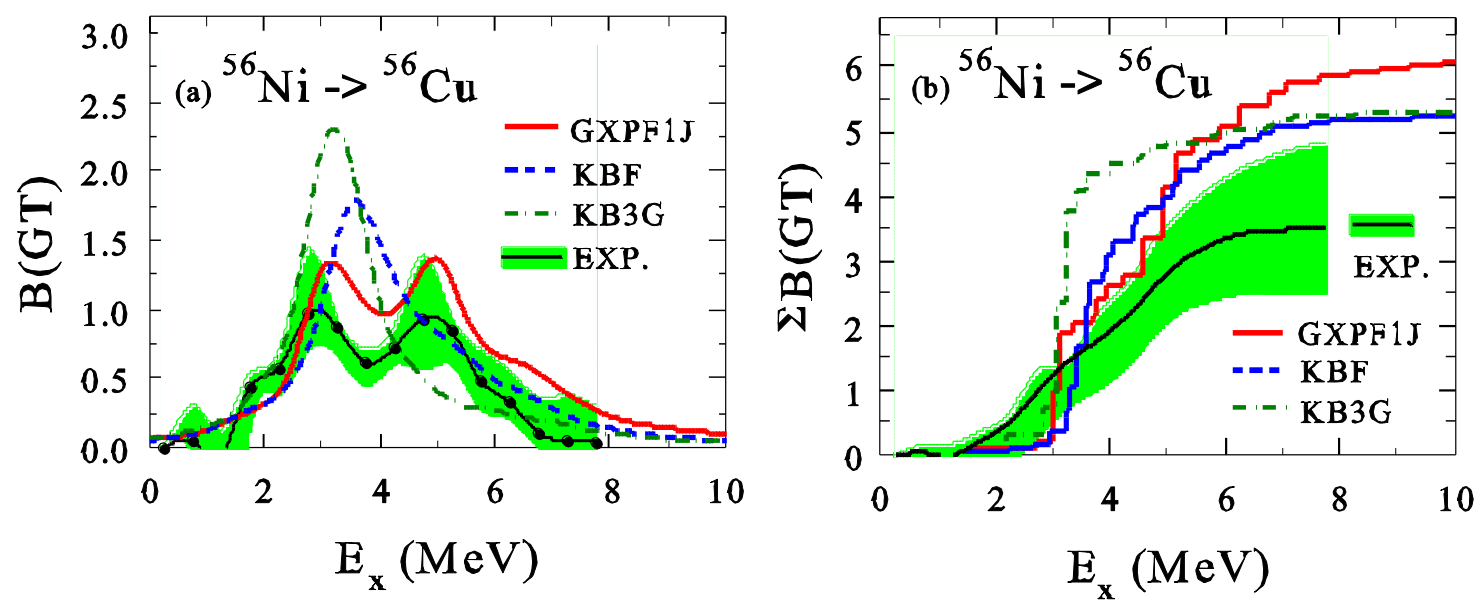

Fig. 1 (a) B(GT.) and (b) cumulative sum of $\mathrm{B}\left(\mathrm{GT}_{\text {- }}\right)$ for ${ }^{56} \mathrm{Ni}$ obtained with GXPF1J, KB3G and KBF Hamiltonians as well as the experimental data

$$
{ }^{56} \mathrm{Ni}\left(\mathrm{e}^{-}, \mathrm{v}\right){ }^{56} \mathrm{Co}
$$

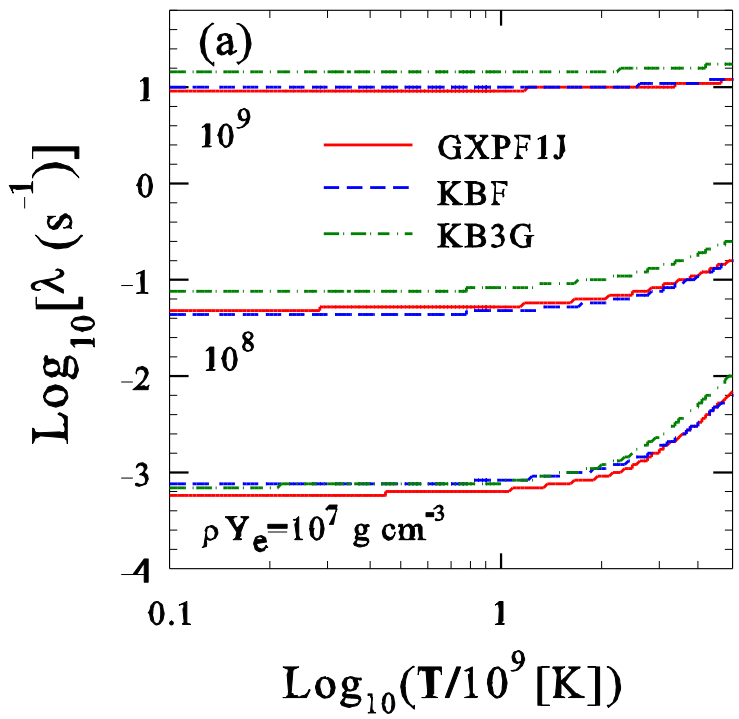

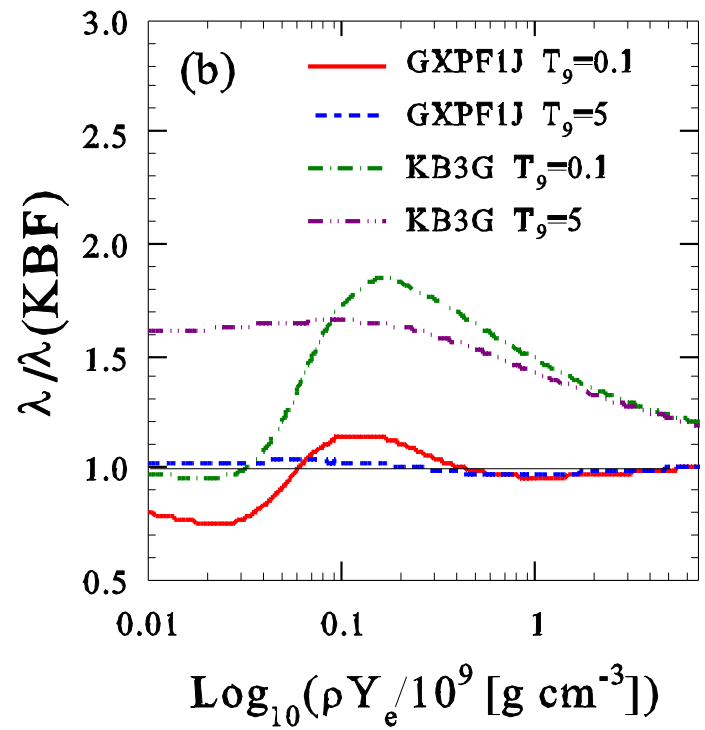

Fig. 2 (a) Electron-capture rates for 56Ni obtained with GXPF1J, KB3G and KBF Hamiltonians. (b) Ratios of the-capture rates of GXPF1J and KB3G over the KBF rates.

\section{Nucleosynthesis of iron-group elements in type Ia supernova explosions}

Accretion of matter to white-dwarfs from their binary stars ignites Type-Ia SNe when the white-dwarf mass approaches the Chandrasekhar limit. A plenty amount of ${ }^{56} \mathrm{Ni}$ is produced in Type-Ia supernova explosions. As e-capture process on ${ }^{56} \mathrm{Ni}$ proceeds, neutron-rich nuclei are produced and $\mathrm{Y}_{\mathrm{e}}$ gets smaller. If e-capture rates on ${ }^{56} \mathrm{Ni}$ are smaller, production yields of neutron-rich isotopes such as ${ }^{58} \mathrm{Ni}$ decreases and $\mathrm{Y}_{\mathrm{e}}$ remains to be a higher value in statistical equilibrium calculations. The ratio of the production yields of ${ }^{58} \mathrm{Ni}$ over ${ }^{56} \mathrm{Ni}$ can be reduced nearly by half for GXPF1J compared to KB3G.

The problem of over-production of ${ }^{58} \mathrm{Ni},{ }^{54} \mathrm{Cr}$ and ${ }^{54} \mathrm{Fe}$ compared to the solar abundance 
was discussed [13] with the use of the capture rates of FFN [1]. A possible solution of the problem with slower e-capture rates was discussed in Ref. [14,15]. The problem could be solved by using further slower e-capture rates of GXPF1J. Here, we use the W7 model [13] as an explosion model of Type-Ia SNe starting from $\mathrm{C}-\mathrm{O}$ white dwarf with the mass of 1.38 solar mass. This model proceeds by a fast deflagration. Electron-capture rates of GXPF1J are used for pfshell nuclei with $21 \leqq Z \leqq 32$ and those of KBF otherwise. The results of final element abundances are shown in Fig. 3. We find that over-production of neutron-rich $\mathrm{Cr}, \mathrm{Fe}$ and $\mathrm{Ni}$ isotopes is suppressed within a factor of 2-3 compared with the solar abundances. This is much smaller than the case of FFN weak rates, where the over-production factor becomes as large as up to 4-5.

The over-production of ${ }^{54} \mathrm{Fe}$ which remained in the W7 model can be improved when we use an explosion model such as WDD2 [13] with slow deflagration and delayed detonation (see right panel of Fig. 3). In this case, the over-production factor is suppressed within twice for all neutron-excess $\mathrm{Cr}$, Fe and $\mathrm{Ni}$ isotopes [16]. Under-production of ${ }^{28} \mathrm{Si},{ }^{32} \mathrm{~S},{ }^{36} \mathrm{Ar}$ and ${ }^{40} \mathrm{Ca}$ is also improved. Thus, the over-production problem of neutron-excess iron-group elements compared with the solar abundances is almost solved though there remains some dependence on the explosion model. The proton-fraction $\mathrm{Y}_{\mathrm{e}}$ is found to be slightly larger for the present rates than the KBF case, but the difference of the element abundances between the present rates and KBF rates is not large; as small as up to $4 \%$. This is due to a cancellation of the difference of the contributions at different densities.
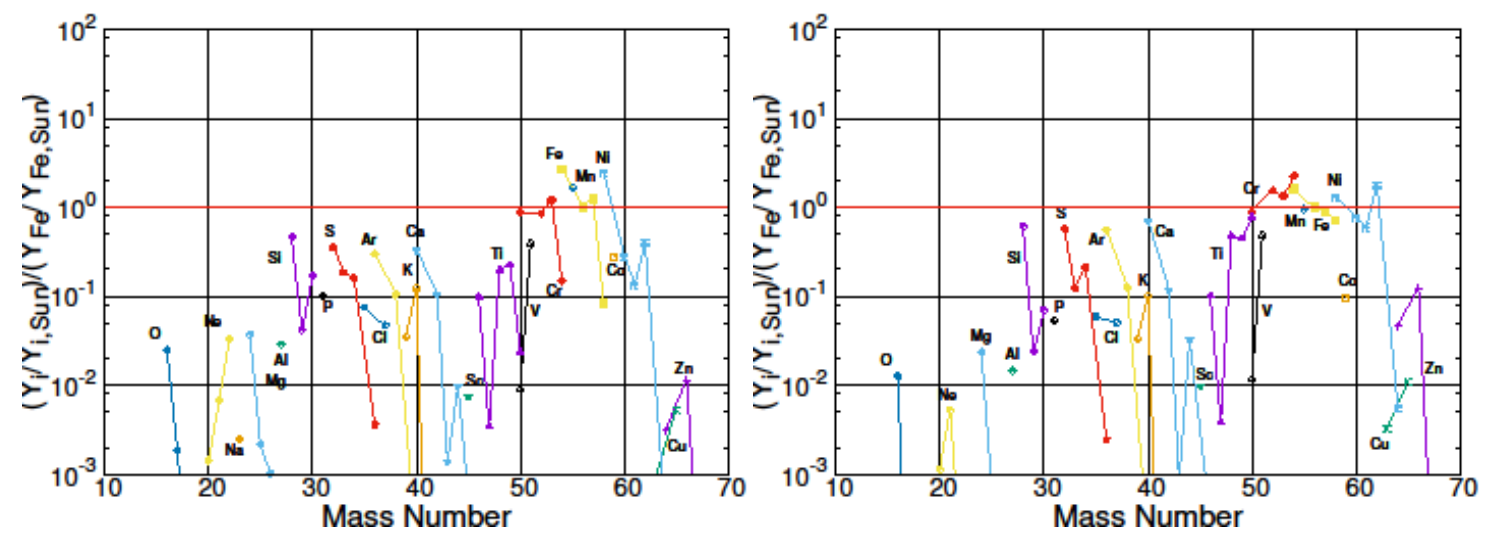

Fig. 3 Ratio of the element abundances compared to the solar abundances obtained with GXPF1J for the W7 explosion model with fast deflagration (left panel) and WDD2 explosion model with slow deflagration and delayed detonation (right panel). (Figures taken from Ref. [16].)

\section{Electron-capture rates in ${ }^{78} \mathrm{Ni}$}

We discuss e-capture rates of ${ }^{78} \mathrm{Ni}$ as one of pf-g shell nuclei which are important for core-collapse SNe. Which nuclei affect the change of $Y_{e}$ most in the core-collapse process? Nuclei with $A=65-105$, especially those along or near the $N=50$ line, are found to have the steepest change of $Y_{e}$ [5]. Evaluation of weak rates in these neutron-rich nuclei is very important, but many of them have not been studied well thus far as extended configurations beyond pfshell are need to be included. For such nuclei, an approximate formula for e-capture rates has been proposed [5].

$$
\begin{aligned}
& \lambda=\frac{\ln 2 \cdot B}{K}\left(\frac{T}{m_{e} c^{2}}\right)^{5}\left[F_{4}(\eta)-2 \chi F_{3}(\eta)+\chi^{2} F_{2}(\eta)\right] \\
& F_{k}(\eta)=\int_{0}^{\infty} \frac{x^{k}}{\exp (x-\eta)+1} d x
\end{aligned}
$$


where $\mathrm{K}=6146 \mathrm{~s} 、 \quad \chi=(\mathrm{Q}-\Delta \mathrm{E}) / \mathrm{T}$ 、 and $\eta=\chi+\mu / \mathrm{T}$ with $\mu$ the electron chemical potential and $\mathrm{T}$ the temperature. Here, $\mathrm{Q}$ is the $\mathrm{Q}$-value for the reaction. Two parameters, effective transition strength $\mathrm{B}$ and shift energy $\Delta \mathrm{E}$ is chosen to be $\mathrm{B}=4.6$ and $\Delta \mathrm{E}=2.5 \mathrm{MeV}$ by fitting to calculated rates obtained by shell-model and RPA [17].

Now, we evaluate electron-capture rates for ${ }^{78} \mathrm{Ni}$ by shell-model and RPA calculations as well as with the approximate formula of Eq. (1), and compare them. Shell-model calculations are performed with the modified A3DA interaction within $\mathrm{pf}-\mathrm{g}_{9 / 2} \mathrm{~d}_{5 / 2}$ configurations including up to $5 \mathrm{p}-5 \mathrm{~h}$ excitations outside the filling configuration of ${ }^{78} \mathrm{Ni}$ [18]. This shell-model calculation gives $\mathrm{E}_{\mathrm{X}}=2.8 \mathrm{MeV}$ for the first $2^{+}$state. The GT, electric-dipole (E1) and spin-dipole (SD) transitions with multipolarities $\lambda^{\mathrm{P}}=0^{-}, 1^{-}$and $2^{-}$are included in the shell-model calculations. RPA calculations are done with SGII interaction [19] including all the multipoles up to $\lambda=4$. Dominant contributions come from SD transitions with $\lambda^{\mathrm{P}}=1^{-}$and $2^{-}$in both cases.

First, GT strength of the inverse transition $\left({ }^{78} \mathrm{Ni} \rightarrow{ }^{78} \mathrm{Cu}\right)$ is studied to compare the various approaches. Cumulative sums of the $\mathrm{B}(\mathrm{GT})$ are shown for shell-model (SM) and RPA calculations in Fig. 4 (left panel). The case of RPA with a truncated configuration including excitations up to pf- $\mathrm{g}_{9 / 2} \mathrm{~d}_{5 / 2}$ orbits is also shown. The quenching factor of $\mathrm{f}_{\mathrm{q}}=\mathrm{g}_{\mathrm{A}}{ }^{\text {eff/ }} \mathrm{g}_{\mathrm{A}}=0.74$ is adopted. The SM exhausts about $54 \%$ of the GT sum-rule value in the present configuration while the RPA exhausts about $80 \%$ of the sum-rule value up to $E_{x}=40 \mathrm{MeV}$. Though the truncated case of RPA exhausts similar amount of GT sum-rule value as the SM, the distribution of the strength is different. The GT strength distribution for the SM calculation is shown in Fig. 4 (right panel), which is spread over several $\mathrm{MeV}$ in contrast to the RPA calculations.
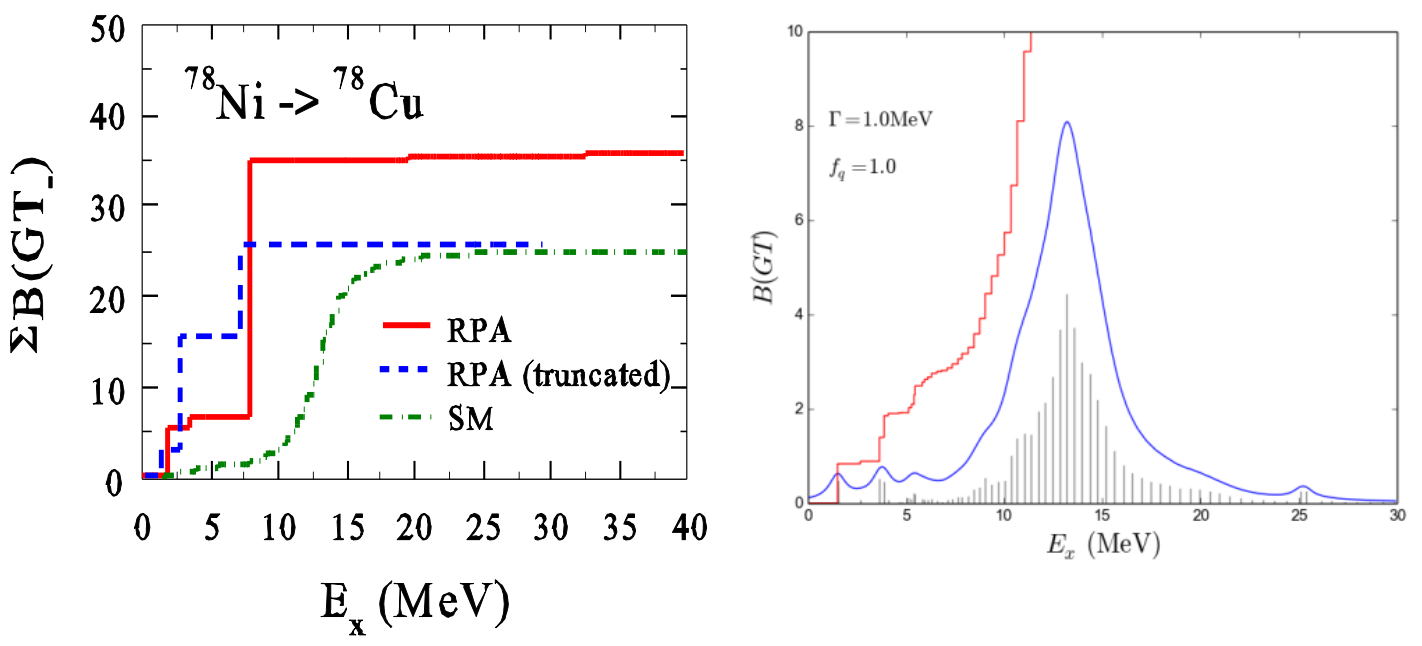

Fig. 4 (Left panel) Cumulative sums of $\mathrm{B}(\mathrm{GT})$ obtained for shell-model (SM), RPA and truncated-RPA calculations. The quenching factor $\mathrm{f}_{\mathrm{q}}=0.74$. (Right panel) The GT strength distribution for the $\mathrm{SM}$ calculation with $\mathrm{f}_{\mathrm{q}}=1.0$. Blue curve is obtained by folding over the Lorenzian with the width of $\Gamma=1 \mathrm{MeV}$. Red curve shows the cumulative sum of $\mathrm{B}(\mathrm{GT})$.

Electron-capture rates obtained by RPA, SM and the approximate formula are compared in Fig. 5. Here, Q-value is taken to be a common value of $\mathrm{Q}=-18.88 \mathrm{MeV}$ [20]. The quenching factor is adopted to be $f_{q}=0.74$ for the GT transition and $f_{q}=1$ for other multipole transitions. The RPA result is found to be rather close to that of the formula Eq. (1). This suggests that Eq. (1) can be considered as a good approximation as long as we use an appropriate Q-value. However, if we use $f_{q}=0.74$ also for other multipoles such as spin-dipole transitions, the difference between RPA and the approximate formula becomes notable. The SM result is found to be much smaller than that of the approximate one, but close to the RPA result with the same truncation (see right panel of Fig. 5). We also find that an RPA result with configurations 
including excitations up to full gds-shell is a very good approximation to the RPA with the fullspace. This suggests that we need to extend the configuration space of SM up to full gds-shell, and also that this extension is good enough.
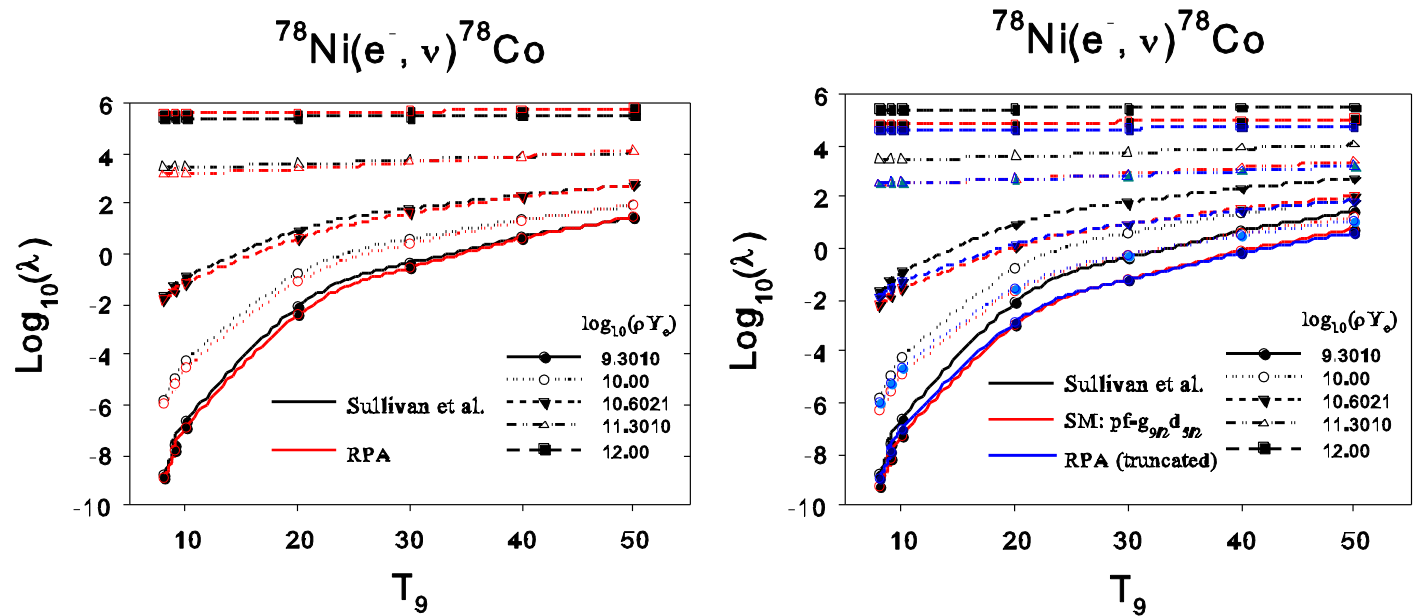

Fig. 5 (Left panel) Electron-capture rates in ${ }^{78} \mathrm{Ni}$ obtained for RPA and the approximate Sullivan's formula Eq. (1). (Right panel) Comparison of calculated electron-capture rates in ${ }^{78} \mathrm{Ni}$ between SM, RPA with the truncated space and Sullivan's formula.

Although SM wave functions are obtained within pf- $\mathrm{g}_{9 / 2} \mathrm{~d}_{5 / 2}$ shell, sum of the strengths of transitions to states with full pf-gds configurations can be obtained. Sum of the strengths of GT, E1 and spin-dipole (SD) transitions,

$$
\mathrm{S}=\sum_{\mathrm{n}}<\text { g.s. }\left|\mathrm{O}^{+}\right| \mathrm{n}><\mathrm{n}|\mathrm{O}| \text { g.s. }>
$$

for $\mathrm{O}(\mathrm{GT})=\vec{\sigma} \mathrm{t}_{+} 、 \mathrm{O}(\mathrm{E} 1)=\mathrm{rY}_{\mathrm{m}}^{(1)} \mathrm{t}_{+} 、 \mathrm{O}(\mathrm{SD} \lambda)=\left[\mathrm{rY} \mathrm{m}_{\mathrm{m}}^{(1)} \times \vec{\sigma}\right]_{\mathrm{m}}^{(\lambda)} \mathrm{t}_{+}$with $\mathrm{t}_{+}|\mathrm{p}>=| \mathrm{n}>$ are given in Table I. For the SM case denoted by SM (pf-gds), the ground state (g.s.) in ${ }^{78} \mathrm{Ni}$ is obtained within the pf- $\mathrm{g}_{9 / 2} \mathrm{~d}_{5 / 2}$ shell but the intermediate states $|\mathrm{n}\rangle$ in Eq. (2) are made by operating the operator $\mathrm{O}$ to the g.s. without any restriction to the $\mathrm{pf}-\mathrm{g}_{9 / 2} \mathrm{~d}_{5 / 2}$ shell. In this way, the final states $|n\rangle$ of the transitions can have extended pf-gds configurations. We see from Table I that the sums of the strengths of SM (pf-gds) are close to the RPA values except for the GT transitions. The inclusion of full gds-shell is necessary for the SM calculation. SM studies with pf-gds shells are now in progress.

\begin{tabular}{|l|l|l|l|}
\hline $\mathrm{O}$ & $\mathrm{SM}\left(\mathrm{pf}-\mathrm{g}_{9 / 2} \mathrm{~d}_{5 / 2}\right)$ & SM (pf-gds) & RPA (full) \\
\hline GT & 0.0078 & 0.0804 & 0.3711 \\
\hline E1 & 3.202 & 4.368 & 4.231 \\
\hline SD0 & 0.046 & 12.083 & 12.378 \\
\hline SD1 & 1.603 & 20.241 & 20.683 \\
\hline SD2 & 2.616 & 14.098 & 15.995 \\
\hline
\end{tabular}

Table I Sums of the strengths of the GT, E1 and SD $\lambda$ transitions (in units of $\mathrm{fm}^{2}$ for E1 and SD transitions) for SM (pf- $\mathrm{g}_{9 / 2} \mathrm{~d}_{5 / 2}$ ) and RPA calculations as well as for SM (pf-gds) case. 
We finally comment on the choice of Q-value and the quenching factor $\mathrm{f}_{\mathrm{q}}$ for spin-dipole transitions. Calculated Q-values in ${ }^{78} \mathrm{Ni}$ are rather scattered, that is, $\mathrm{Q}=-18.1 \mathrm{MeV}$ with a Coulomb correction for the SM [18], Q = -16.29 MeV and -18.88 MeV for RPA with SGII [19] and HFB21 [20], respectively, and $\mathrm{Q}=-21.19 \mathrm{MeV}$ for FRDM [21]. As the capture rates depend sensitively on the Q-value, it is important to choose a reliable one. The shell-model calculations are promising to obtain reliable Q-values. We have not enough information on the quenching factor in spin-dipole transitions. This problem is left to future investigations.

\section{Summary}

GT strengths in $\mathrm{Ni}$ and $\mathrm{Fe}$ isotopes have been studied with a new shell-model Hamiltonian in pf-shell, GXPF1J. They are found to be more spread compared to those of $\mathrm{KB} 3 \mathrm{G}$ and $\mathrm{KBF}$, and consistent with recent experimental data, especially in ${ }^{56} \mathrm{Ni}$. Electroncapture rates in $\mathrm{Ni}$ isotopes, iron-group nuclei and pf-shell nuclei are evaluated with GXPF1J at stellar environments, and applied to nucleosynthesis in Type Ia SNe. GXPF1J gives smaller ecapture rates compared with KB3G, FFN and KBF (in case of KBF it depends on the density if the rates are smaller or larger), and leads to larger $Y_{e}$ with less production of neutron-rich isotopes. The over-production problem of neutron-excess iron-group elements with the FFN rates can thus be solved by using the present accurate capture rates.

Electron-capture rates for ${ }^{78} \mathrm{Ni}$ are evaluated by shell-model and RPA calculations, and compared with the approximate Sullivan's formula. The capture rates obtained by RPA are found to be close to those of Sullivan's if the quenching factor $f_{q}=g_{A}$ eff $/ g_{A}$ is taken to be 1.0 for the spin-dipole transitions. The shell-model calculations with the present $\mathrm{pf}-\mathrm{g}_{9 / 2} \mathrm{~d}_{5 / 2}$ shell is not good enough for the spin-dipole transitions, and the extension of the space to fp-gds configurations is necessary. Studies with extension of the shell-model space are now under way. Precise Q-value in the transition is important for reliable evaluations of the capture rates, and shell-model would be promising to obtain more accurate energies of low-lying states.

This work has been supported in part by Grants-in-Aid for Scientific Research (C) 15K05090 and JSPS (26105517 and 24340060) of the MEXT of Japan, as well as by NSF grant PHY1430152 and PHY-1204486, and by an NAOJ Visiting Research Professorship. It was supported also in part by Priority Isuue on post-K computer (hp160211) and CNS-RIKEN joint project for large-scale nuclear structure calculations.

\section{References}

[1] G. M. Fuller, W. A. Fowler and M. J. Newman, Stellar weak interaction rates for intermediate-mass nuclei. $I I-A=21$ to $A=60$, The Astrophy. J. 252 (1982) 715.

[2] M. Honma et al., Effective interaction for pf-shell nuclei, Phys. Rev. C 65 (2002) 061301 (R);

M. Honma et al., Effective interaction for nuclei of A=50-100 and Gamow-Teller properties, J. Phys. Conf. Ser. 20 (2005) 7.

[3] T. Suzuki, M. Honma, H. Mao, T. Otsuka and T. Kajino, Evaluation of electron capture reaction rates in Ni isotopes in stellar environments, Phys. Rev. C 83 (2011) 044619. 
[4] T. Suzuki and T. Kajino, Element synthesis in the supernova environment and neutrino oscillations, J. Phys. G 40 (2013) 083101.

[5] C. Sullivan, E. O’Connor, R. G. T. Zegres, T. Grubb and S. M. Austin, The sensitivity of corecollapse supernovae to nuclear electron capture, The Astrophys. J. 816 (2016) 44.

[6] E. Caurier, G. Martinez-Pinedo, F. Nowacki, A. Poves and A. P. Zuker, The shell model as a unified view of nuclear structure, Rev. Mod. Phys. 77 (2005) 427.

[7] G. Martinez-Pinedo, A. Poves, E. Caurier and A. P. Zuker, Effective $g_{A}$ in the pf shell, Phys. Rev. C 53 (1996) R2602.

[8] M. Honma, T. Otsuka, B. A. Brown and T. Mizusaki, Shell-model description of neutron-rich pfshell nuclei with a new effevtive interaction GXPF1, Eur. Phys. J. A 25 , Suppl. 1 (2005) 499.

[9] Y. Fujita, B. Rubio and W. Gelletry, Spin-isospin excitations probed by strong, weak and electromagnetic intearctions, Prog. Part. Nucl. Phys. C 66 (2011) 549.

[10] A. L. Cole et al., Gamow-Teller strengths and electron-capture rates for pf-shell nuclei of relenance for late stellar evolution, Phys. Rev. C 86 (2012) 015809.

[11] E. Caurier, K. Langanke, G. Martinez-Pinedo and F. Nowacki, Shell-model calculations of stellar weak interaction rates. 1. Gamow-Teller distributions and spectra of nuclei in the mass range $A=$ 45-65, Nucl. Phys. A 653 (1999) 439.

[12] M. Sasano et al., Gamow-Teller Transition Strengths from ${ }^{56}$ Ni, Phys. Rev. Lett. 107 (2011) 202501; M. Sasano et al., Extraction of Gamow-Teller strength distributions from ${ }^{56} \mathrm{Ni}$ and ${ }^{55} \mathrm{Co}$ via the $(p, n)$ reaction, Phys. Rev. C 86 (2012) 034324.

[13] K. Iwamoto et al., Nucleosynthesis in Chandrasekahar mass models for Type Ia supernovae and constraints on progenitor systems and burning-front propagation, The Astrophys. J. 125 (1999) 439.

[14] F. Brachwitz et al., The role of electron captures in Chandrasekahar-mass models for Type Ia supernovae, The Astrophys. J. 536 (2000) 934.

[15] K. Langanke and G. Martinez-Pinedo, Nuclear weak-interaction processes in stars, Rev. Mod. Phys. 75 (2003) 819.

[16] K. Mori, M. A. Famiano, T. Kajino, T. Suzuki, J. Hidaka, M. Honma, K. Iwamoto, K. Nomoto and T. Otsuka, Impact of new Gamow-Teller strengths on explosive Type Ia supernova nucleosynthesis, The Astrophys. J. 833 (2016) 179.

[17] K. Langanke et al., Electron Capture Rates on Nuclei and Implications for Stellar Core Collapse, Phy. Rev. Lett. 90 (2003) 241102.

[18] Y. Tsunoda, T. Otsuka, N. Shimizu, M. Honma and Y. Utsuno, Novel shape evolution in exotic Ni isotopes and configuration-dependent shell structure, Phys. Rev. C 89 (2014) 031301(R).

[19] N. Van Giai and H. Sagawa, Spin-isospin and pairing properties of modified Skyrme interactions, Phys. Lett. B 106 (1981) 379.

[20] S. Goriely, N. Chamel and J. M. Pearson, Further explorations of Skyrme-Hartree-Fock-Bogoliubov mass formulas. XII. Stiffness and stability of neutron-star matter, Phys. Rev. C 82 (2010) 035804.

[21] P. Moller, J. R. Nix and K.-L. Kratz, Nuclear properties for astrophysical and radioactive-ion-beam applications, At. Data and Nucl. Data Tables 66 (1997) 131. 